\title{
Dealing with Uncertainties in Fatigue Strength Using Deep Rolling
}

\author{
Berkay Yüksel $^{1,2(\bowtie)}$ (D) and Mehmet Okan Görtan ${ }^{1,2}$ (D) \\ ${ }^{1}$ Hacettepe University, 06800 Ankara, Turkey \\ berkay.yuksel@hacettepe.edu.tr \\ 2 National Nanotechnology Research Center (UNAM), Bilkent University, \\ 06800 Ankara, Turkey
}

\begin{abstract}
Mechanical properties inherently possess uncertainties. Among these properties, fatigue behavior data generally shows significant scatter which introduces a challenge in the safe design of dynamically loaded components. These uncertainties in fatigue behavior are mainly results of factors related to surface state including: Roughness, tensile residual stresses, scratches and notches at surface. Therefore, controlling these parameters allows one to increase fatigue strength and reduce scatter and uncertainties in fatigue behavior. Mechanical surface treatments are applied on parts to increase fatigue strength via introducing compressive residual stresses and work-hardening at surface. Two of the most common among these treatments are shot peening and deep rolling. Shot peening has found many applications in industry because of its flexibility. However, it introduces irregularities at the surface and may increase roughness which causes uncertainties in the fatigue behavior data; especially for low-medium strength materials. Unlike shot peening, deep rolling reduces surface roughness. Therefore, it has the capability to reduce uncertainty in the fatigue behavior. To this date, rolling direction of deep rolling was selected as tangential direction to turning direction for axisymmetric parts. Nonetheless, the authors believe that the rolling direction has an apparent effect on the fatigue behavior. In this study, longitudinal direction was also applied for deep rolling operation and the results of these two direction applications on the EN-AW-6082 aluminum alloy were investigated. It was shown that, longitudinal rolling had yielded less scatter and uncertainty in the fatigue behavior than the tangential rolling together with the higher fatigue strength.
\end{abstract}

Keywords: Deep rolling · Fatigue behavior $\cdot$ Aluminum alloy

\section{Introduction}

Due to the tightening in regulations on emission standards [1], usage of lightweight materials like aluminum alloys has increased over the years in the automotive industry. One of the most commonly used aluminum alloys are the heat-treatable 6xxx series owing to their strength-to-weight ratio, formability characteristics and corrosion resistance $[2,3] .6 x x x$ series alloys are commonly used for the design of critical parts which are subjected to dynamic loading like suspension parts [4]. 
Mechanical surface treatments are commonly applied on the parts which are dynamically loaded in order to increase their fatigue strength properties. Among these treatments shot peening and deep rolling are the most commonly applied ones [5]. These treatments are applied to induce local plastic deformation on the parts which causes favorable compressive residual stresses and a work-hardened layer at the surface region. Shot peening is applied via peening the part using steel or ceramic balls with a pre-determined intensity and time whereas deep rolling is applied using hydraulically or mechanically supported roller or a ball to deform the material surface with a well-defined force. Although the compressive residual stresses induced by deep rolling generally reaches higher depths than shot peening [6], shot peening process has been a more investigated subject compared to deep rolling process to this date owing to its flexibility and ease of application. However, shot peening inherently has a negative effect on the surface roughness parameters due to its principles [5]. Low-to-medium strength materials are especially prone to these negative effects and if the severity of the shot peening is not chosen correctly, process may induce crack-like irregularities on the material surface $[5,7,8]$. On the other hand, deep rolling reduces surface roughness if applied with correct forces [5, 9]. Reduced surface roughness is known to be beneficial for fatigue strength properties. Because of these reasons deep rolling stands out to be an attractive option for the critical parts which are to be manufactured from 6xxx series alloys in automotive industry.

Although improvement can be made via surface treatments, fatigue behavior data generally shows significant scatter and uncertainty. Therefore, it should be subjected to statistical methods in order to overcome these uncertainties to allow safe design of the parts. Within engineering community, lower 2-sigma or 3-sigma design curves commonly employed. However, these methods fail to account for providing the definite confidence intervals and reliability levels [10]. On the other hand, Owen one-side tolerance limit method is a viable option in order to determine the confidence and reliability levels [11]. In both of these methods, standard error of the specimen set about the median curve is used and assumed to be uniform for the entire range of the data set. For lower 3sigma approach, standard error is multiplied by three and algebraically subtracted from the least-squares curve to obtain the lower limit curve. For the Owen one-side tolerance limit, coefficient $\mathrm{K}_{\text {owen }}$ is found by employing empirical coefficients and multiplied with the standard error $[10,11]$. Different $\mathrm{K}_{\text {owen }}$ values corresponding to various confidence and reliability levels were tabulated in [12].

In this study, commercial EN-AW 6082 aluminum alloy specimens were subjected to deep rolling to improve fatigue strength properties. Although deep rolling is conventionally applied in the tangential direction for axisymmetric parts, rolling in the longitudinal direction was also applied. Effects of rolling direction on fatigue strength and its uncertainty were investigated and the results were compared with untreated specimens. Statistical approaches were used to evaluate safe design ranges for fatigue strength. Both lower-3 sigma design curves and Owen one-side tolerance limits were considered and compared.

\section{Materials and Methods}

For this study, EN-AW 6082 aluminum bars of $1 \mathrm{~m}$ length and $15 \mathrm{~mm}$ diameter were supplied. Chemical composition of the material was shown in Table 1. From these 
bars specimens of $120 \mathrm{~mm}$ length were cut and then solution heat-treated at $550{ }^{\circ} \mathrm{C}$ for $1.5 \mathrm{~h}$, quenched and artificially aged at $180{ }^{\circ} \mathrm{C}$ for $8 \mathrm{~h}$. After the heat treatment procedure, both tensile and fatigue test specimens were machined to their respective geometries using Spinner TC600-35 CNC turning machine with $0.4 \mathrm{~mm}$ depth of cut and $0.105 \mathrm{~mm} /$ revolution feed rate. Finishing cut was done using $0.1 \mathrm{~mm}$ depth of cut.

Table 1. Chemical composition of 6082 aluminum alloy

\begin{tabular}{l|l|l|l|l|l|l}
\hline $\mathrm{Al}$ & $\mathrm{Si}$ & $\mathrm{Fe}$ & $\mathrm{Cu}$ & $\mathrm{Mn}$ & $\mathrm{Mg}$ & $\mathrm{Cr}$ \\
\hline 97.2 & 0.93 & 0.295 & 0.015 & 0.53 & 1.00 & 0.0055 \\
\hline
\end{tabular}

Mechanical properties of the alloy were determined using tensile tests. In these tests, specimens of Type A in accordance with DIN 50125: 2009-07 standard were used. Specimens had $40 \mathrm{~mm}$ gauge length and $8 \mathrm{~mm}$ diameter and the strain rate was selected as $1 \times 10^{-3}$. Yield point was determined using $0.2 \%$ offset rule. Mechanical properties were averaged from the data of 3 specimens. Apart from these tests, Vickers microhardness measurements were done on the section perpendicular to the longitudinal axis of the specimens using $500 \mathrm{~g}$ force and $10 \mathrm{~s}$ dwell time. Mechanical properties of the alloy were summarized in Table 2 .

Table 2. Mechanical properties of 6082 aluminum alloy

\begin{tabular}{l|l|l|l}
\hline Yield strength $[\mathrm{MPa}]$ & Tensile strength $[\mathrm{MPa}]$ & Elongation at fracture [\%] & HV0.5 \\
\hline $280.8 \pm 5.9$ & $312.0 \pm 9.8$ & $22.3 \pm 1.4$ & $123.1 \pm 3.1$ \\
\hline
\end{tabular}

Deep rolling process was applied on the specimens in two different directions: Tangential (conventional) and longitudinal directions. These directions can be seen in Fig. 1. For both directions, $250 \mathrm{~N}$ of rolling force was used and feed was selected as $0.1 \mathrm{~mm} /$ pass. Rolling speed was selected as $10 \mathrm{~mm} / \mathrm{s}$ for both processes. Roller radius and tip radius of the deep rolling apparatus were $42.5 \mathrm{~mm}$ and $2.5 \mathrm{~mm}$, respectively. Using Hertz Theory; maximum contact pressures were calculated as $2610 \mathrm{MPa}$ and $1750 \mathrm{MPa}$ for tangentially rolled (TR) and longitudinally rolled (LR) cases, respectively.

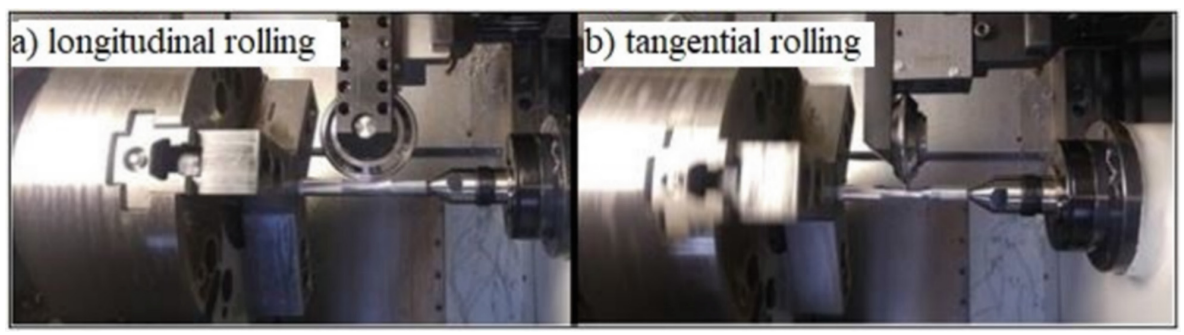

Fig. 1. Deep rolling directions 
Surface roughness parameters of $\mathrm{Ra}$ and $\mathrm{Rz}$ were determined using Mitutoyo SJ210 profilometer with skidded stylus tip. Average of 10 measurements were used for each set in accordance with ISO 4287-1994 standard and in the direction parallel to the longitudinal direction of the specimen. Since the apparatus was skidded, roughness measurement in the tangential direction was not possible because of the measurement errors associated with the specimen curvature. Measurement section can be seen in Fig. 2.

Deep rolling induced work-hardening state of the material surface was qualitatively determined via Vickers micro hardness measurements close to the surface with $10 \mathrm{~g}$ force and $10 \mathrm{~s}$ dwell time. Measurements were started from the near-surface region and proceeded towards the bulk of the material in order to be able to observe the differences between near-surface region and bulk of the material.

Residual stresses at the surface were measured by using X'Pert PANalytical multipurpose X-ray diffractometer at National Nanotechnology Research Center (UNAM). Peak location for $\mathrm{CuK} \alpha$ radiation of $2 \theta=138^{\circ}$ were used and 9 different tilt angles were measured between $-39.25^{\circ}<\psi<39.25^{\circ}$. Residual stress measurements were done on fatigue specimens. Diffraction area on the specimen surface was a circle with an approximate diameter of $2 \mathrm{~mm}$ in order to minimize the errors associated with the curvature of the specimens. Measurement section can be seen in Fig. 2.

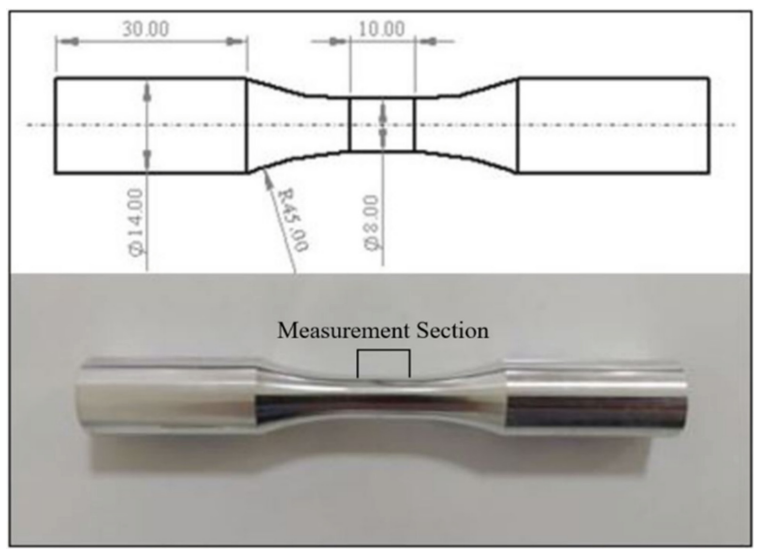

Fig. 2. Fatigue test specimen geometry

Fatigue tests were conducted as force-controlled constant amplitude tests by employing servo-hydraulic axial fatigue testing machine with $4 \mathrm{~Hz}$ frequency and load ratio of $\mathrm{R}=-1$. In these tests specimens with gage length of $10 \mathrm{~mm}$ and diameter of $8 \mathrm{~mm}$ were used. Specimen can be seen in Fig. 2. Since high cycle fatigue is technologically more important in automotive industry, tests were done at stress levels that corresponds to cycle counts that are above $10^{4} .12$ specimens for each case were tested for untreated (UT) tangentially rolled (TR) and longitudinally rolled (LR) cases with the total of 36 specimens. After the tests were finished, obtained fatigue behavior data were subjected to lower 3-sigma method and Owen one-side tolerance limit method with $90 \%$ reliability and 90\% confidence (R90C90). This way, practical limits which allow for the safe design were determined. 


\section{Results and Discussions}

Roughness alteration after deep rolling process was shown in Table 3. Both TR and LR specimen sets showed similar improvements for roughness parameters Ra and Rz. Ra values dropped from approximately $0.7 \mu \mathrm{m}$ to $0.1 \mu \mathrm{m}$; whereas $\mathrm{Rz}$ values dropped from approximately $2.8 \mu \mathrm{m}$ to $0.5 \mu \mathrm{m}$. These improvements on the roughness values has a beneficial effect on the fatigue behavior since surface irregularities act as stress concentrators and crack nucleation sites. However, it is important to note that roughness after deep rolling is strongly dependent on the roughness prior to deep rolling [9].

Table 3. Roughness parameters

\begin{tabular}{l|l|l}
\hline & $\operatorname{Ra}[\mu \mathrm{m}]$ & $\operatorname{Rz}[\mu \mathrm{m}]$ \\
\hline UT & $0.720 \pm 0.010$ & $2.797 \pm 0.128$ \\
\hline TR & $0.092 \pm 0.007$ & $0.542 \pm 0.103$ \\
\hline LR & $0.107 \pm 0.018$ & $0.535 \pm 0.072$ \\
\hline
\end{tabular}

Figure 3 demonstrates the roughness profiles of LR and TR specimen. Even though the obtained numerical values for $\mathrm{Ra}$ and $\mathrm{Rz}$ were similar, there were significant differences in the roughness profiles. The regions marked with grey ellipses for TR specimen's roughness profile exhibited significantly higher valley depths compared to the other regions which may act as crack nucleation sites. Therefore, these regions may

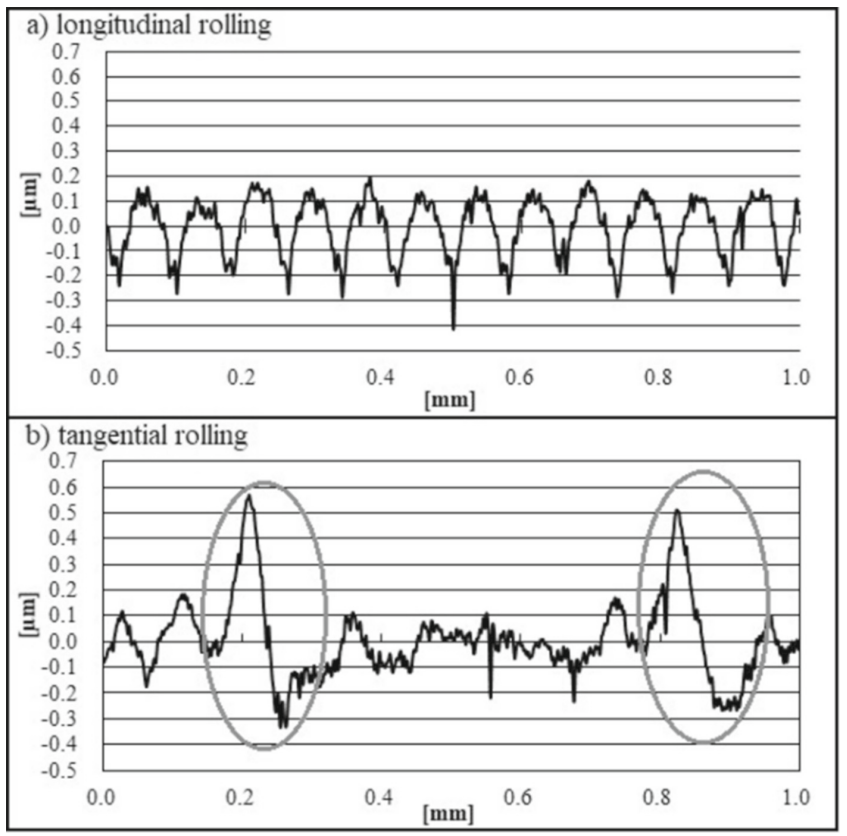

Fig. 3. Roughness profiles 
increase probability of the crack nucleation and increase the uncertainties in the fatigue behavior data.

Hardness distribution within the material in the depth direction can be seen in Fig. 4 . Solid line on the figure represents the nominal untreated material hardness value of 123.1 HV. Increase in the hardness is associated with the work-hardening. Therefore, plastic deformation depth induced by deep rolling process can be assessed. It can be seen that, for both rolling directions, plastic deformation depth reached up to nearly 0.5-0.6 mm. Hardness increase usually retards crack initiation; therefore, is expected to have a positive effect on the fatigue behavior. Near surface, hardness increase was approximately $10 \%$.

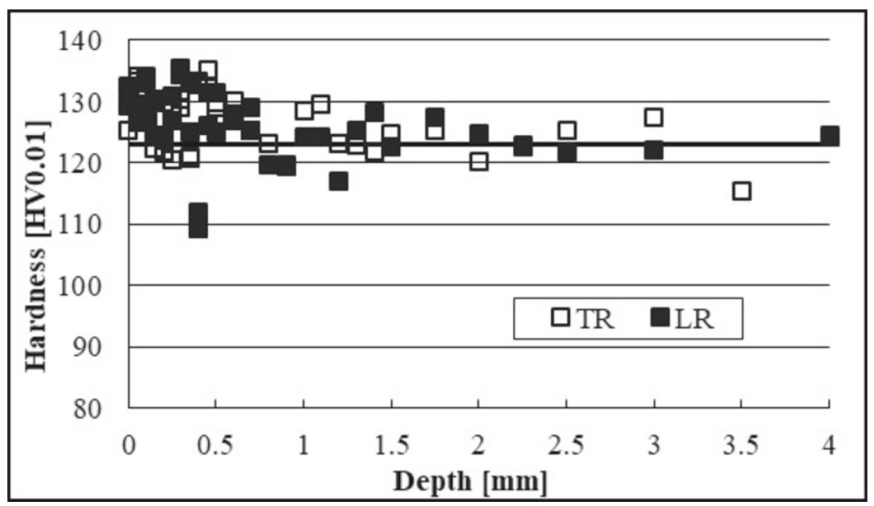

Fig. 4. Hardness distribution after deep rolling

Measured residual stress values at the surface that were developed after deep rolling operation can be seen in Table 4. For both rolling direction, residual stresses in longitudinal $(\sigma \mathrm{z})$ and tangential directions $(\sigma \mathrm{x})$ had a negative value; which is beneficial for fatigue strength improvement. It can be observed that the residual stresses in the tangential direction had higher compressive stress values for LR specimens compared to TR. This is favorable to hinder the crack propagation and expected to contribute to fatigue strength improvement of LR specimens more than TR ones. On the other hand, difference between residual stresses in longitudinal (loading) direction is relatively small and negligible.

Table 4. Residual stress measurements after the deep rolling operation

\begin{tabular}{l|l|l}
\hline & $\sigma z[\mathrm{MPa}]$ & $\sigma \mathrm{x}[\mathrm{MPa}]$ \\
\hline TR & $-189.9 \pm 13.3$ & $-98.1 \pm 15.8$ \\
\hline LR & $-178.3 \pm 18.5$ & $-190.4 \pm 20.0$ \\
\hline
\end{tabular}

Fatigue life behavior of UT, TR and LR specimens can be seen in Fig. 5. Note that the drawn curves are least-squares regression curves in semi-log coordinates and represents 
the median curves for the fatigue life. It can be clearly seen that both deep rolling operations improved high cycle fatigue behavior. Although tangential rolling resulted in only a slight improvement, longitudinal rolling had a significantly pronounced positive effect on the fatigue behavior. For the materials which do not exhibit a well-defined endurance limit, stress amplitude level at $10^{6}$ cycles is a commonly adopted value in order to be able to quantify the fatigue strength of the material. Median curve of the UT specimen set indicated the fatigue strength of approximately $136 \mathrm{MPa}$ at $10^{6}$ cycles; whereas for the TR and LR specimen sets, this value increased to $145 \mathrm{MPa}$ and $167 \mathrm{MPa}$, respectively.

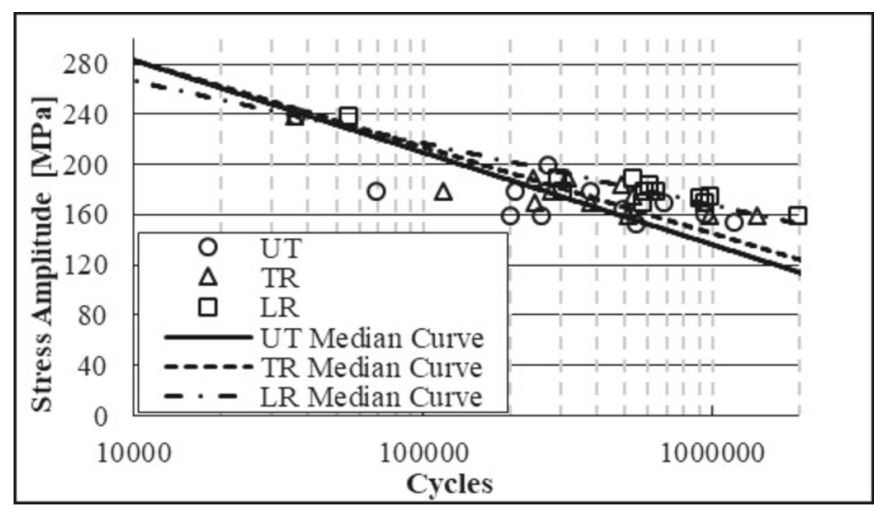

Fig. 5. Fatigue behavior of UT, TR and LR specimen sets

Although median fatigue curves are useful in order to make comparison between different sets, they often possess significant amount of scatter and uncertainty associated with this scatter as seen from the Fig. 5. Figure 6, 7 and 8 shows the lower 3-sigma and the R90C90 Owen one-sided limits together with the median curves for UT, TR and LR sets, respectively.

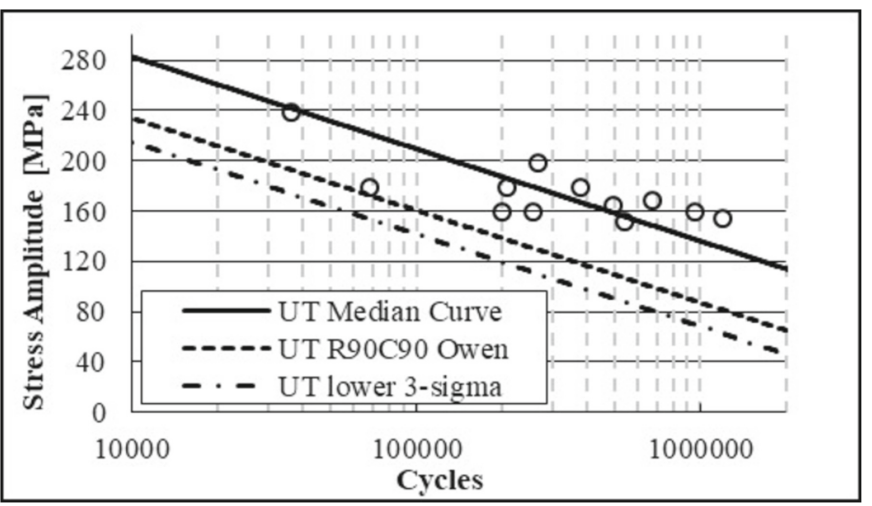

Fig. 6. Fatigue behavior and lower bounds of the UT specimen set 


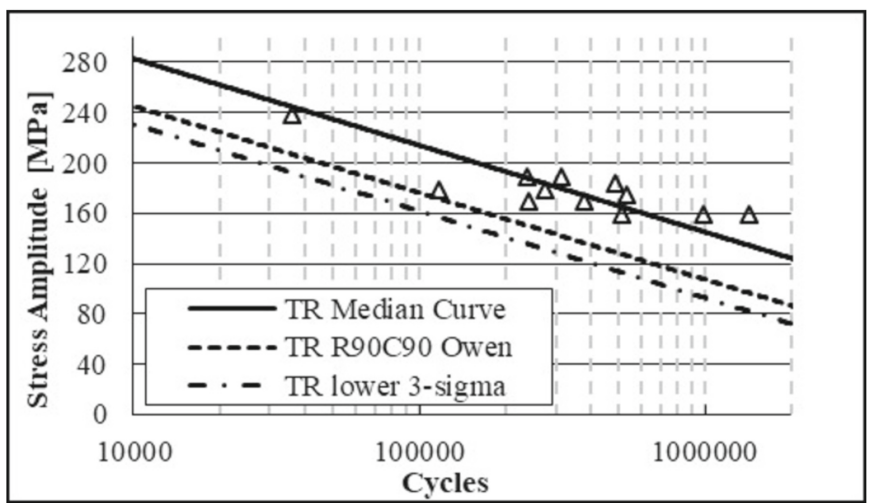

Fig. 7. Fatigue behavior and lower bounds of the TR specimen set

As seen in the Fig. 6, 7 and 8, difference between lower bounds and the median curve was maximum for UT specimen set and minimum for the LR specimen set. This difference is due the scatter within the data and increases as the scatter increases.

Fatigue strength for the R90C90 Owen curves at $10^{6}$ cycles were $87 \mathrm{MPa}, 107 \mathrm{MPa}$ and $150 \mathrm{MPa}$ for UT, TR and LR specimen sets, respectively. For the lower 3-sigma curves, these values found to be $68 \mathrm{MPa}, 93 \mathrm{MPa}$ and $144 \mathrm{MPa}$ for UT, TR and LR sets, respectively. As can be seen, lower-3 sigma curve was more conservative than the R90C90 Owen curve for this sample size; meaning it had higher confidence and reliability level. However, care should be taken to make a conclusion as the $\mathrm{K}_{\text {owen }}$ values change with the sample size. Therefore, if the lower sigma approaches are to be used, it can be a better practice to use lower- 2 sigma rather than lower- 3 sigma for the large sample sizes (above 30 samples). Because, $\mathrm{K}_{\text {owen }}$ values decreases as the sample size increases due to the reduction in the uncertainties. In that case, use of the lower- 3 sigma curve may cause excessive material usage and increase the weight of the structure.

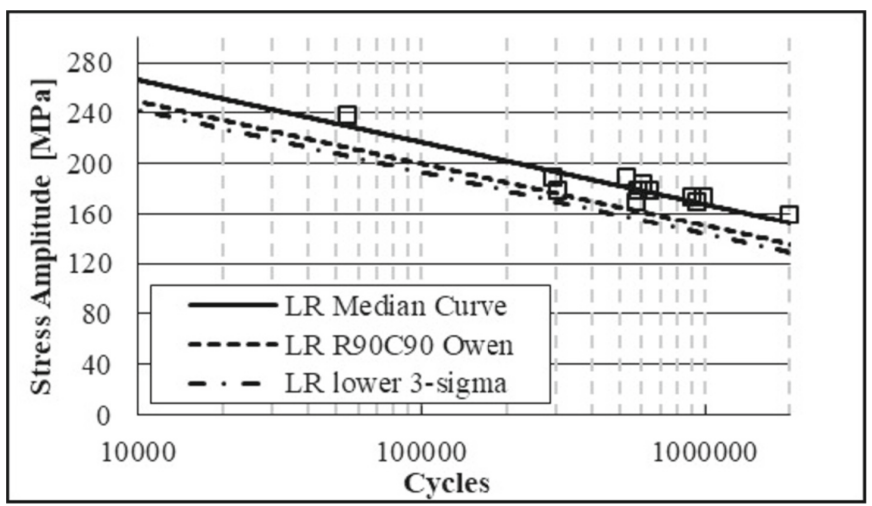

Fig. 8. Fatigue behavior and lower limits of LR specimen set 
Improvement of fatigue strength at $10^{6}$ cycles in percentage were given in the Table 5 . Since the UT median curve possessed higher level of uncertainties compared to the TR and LR sets, improvement in the fatigue strength after the deep rolling became more pronounced with the statistical treatment applied on the experimental data set. It can also be seen that LR yielded significantly higher fatigue strength and less scatter than the TR. This behavioral difference between rolling direction is believed to be mostly related to geometrical alterations at the surface. Because, residual stresses were expected to have similar values for all specimens within each set. Therefore, uncertainty difference between TR and LR set could not be explained by residual stresses only.

Table 5. Fatigue strength improvements [\%]

\begin{tabular}{c|l|l|l}
\hline & Median curve & R90C90 owen & $\begin{array}{l}\text { Lower } \\
\text { 3-Sigma }\end{array}$ \\
\hline TR & 6.6 & 23.0 & 36.8 \\
\hline LR & 22.8 & 72.4 & 111.8 \\
\hline
\end{tabular}

Differences between TR and LR sets in terms of experimental fatigue data were mainly attributed to the direction of the grooves formed by deep-rolling operation and the differences in the roughness profiles. Authors acknowledge the fact that for LR, roughness profile would look similar to the profile shown in Fig. 3b if the measurement were done in the tangential direction. However, it was stated in [13] that polishing in which the fine scratches oriented parallel to the loading direction had yielded better results than perpendicular direction in terms of fatigue strength. Analogous to that phenomenon it can be expected that the grooves/ridges parallel to the loading direction would result in consistently higher fatigue strength than the perpendicular one as the latter case may promote crack nucleation. In addition to that, the aforementioned differences in the roughness profiles in the longitudinal (loading) direction are believed to be one of the major reasons of the lower fatigue strength and higher uncertainty of the TR specimen set compared to LR. Since the effects of the longitudinal rolling direction compared to tangential direction were not investigated in the literature before; differences between LR and TR sets mentioned above are of technological importance.

\section{Conclusions and Recommendations}

It can be seen in the above results that the fatigue strength determination is prone to uncertainties. Because of these uncertainties, usage of lower-bound curves are essential for safe design. In this study, beneficial effects of the deep rolling in different rolling directions on surface properties and fatigue behavior of the EN-AW 6082-T6 aluminum alloy were shown. These effects can be listed as below;

- Although surface roughness values were significantly reduced to similar values for both rolling directions, there were significant differences between profiles. TR specimen set had more inconsistent roughness profile and deeper valleys than LR set. 
- Hardness values showed increased values near-surface region after deep rolling because of the work-hardening behavior without a significant difference between TR and LR specimen sets.

- Favorable compressive residual stresses formed at the surface after deep rolling.

- Fatigue strength properties were improved after deep-rolling compared to the untreated parts. This improvement was significantly more pronounced for the longitudinal rolling than the tangential rolling.

- For the untreated specimen set, scatter within the data and the uncertainties associated with this scatter was found to be highest among the specimen sets. Because of this, fatigue strength improvements due to deep rolling operation became even more pronounced after the statistical treatment of the median curves.

- Longitudinal rolling yielded less scatter and uncertainties than the tangential rolling.

In the future, reach of this study can be extended to include different deep rolling parameters to acquire more comprehensive knowledge on the material and the process.

Acknowledgments. The authors thank the Scientific and Technological Research Council of Turkey (TÜBITTAK) for financial support (Project Number: 217M962)

\section{References}

1. Advanced High-Strength Steel Applications Guidelines 6.0. World Steel Association. http://www.worldautosteel.org/projects/advanced-high-strength-steel-application-gui delines/. Accessed 14 Aug 2017

2. Aruga, Y., Kozuka, M., Takaki, Y., Sato, T.: Effects of natural aging after pre-aging on clustering and bake-hardening behavior in an Al-Mg-Si alloy. Scripta Mater. 116(1), 82-86 (2016)

3. Abdulstaar, M., Mhaede, M., Wagner, L.: Pre-corrosion and surface treatments effects on the fatigue life of AA6082 T6. Adv. Eng. Mater. 15(10), 1002-1006 (2013)

4. Jeswiet, J., Geiger, M., Engel, U., Kleiner, M., Schikorra, M., Duflou, J., Neugebauer, R., Bariani, P., Bruschi, S.: Metal forming progress since 2000. CIRP J. Manufact. Sci. Technol. 1, 2-17 (2008)

5. Schulze, V.: Modern Mechanical Surface Treatments, 1st edn. WILEY-VCH Verlag Gmbh \& Co. KGaA, Weinheim (2006)

6. Sticchi, M., Schnubel, D., Kashaev, N., Huber, N.: Review of residual stress modification techniques for extending the fatigue life of metallic components. Appl. Mech. Rev. 67(1), 1-9 (2015)

7. Trsko, L., Guagliano, M., Bokuvka, O., Novy, F.: Fatigue life of AW 7075 aluminum alloy after severe shot peening treatment with different intensities. Procedia Eng. 74(1), 246-252 (2014)

8. Gonzalez, J., Bagherifard, S., Guagliano, M., Pariente, I.F.: Influence of different shot peening treatments on the surface state and fatigue behaviour of Al 6063 alloy. Eng. Fract. Mech. 185(1), 72-81 (2017)

9. Beghini, M., Bertini, L., Monelli, B.D., Santus, C., Bandini, M.: Experimental parameter sensitivity analysis of residual stresses induced by deep rolling on 7075-T6 aluminium alloy. Surf. Coat. Technol. 254(1), 175-186 (2014) 
10. Lee, Y., Pan, J., Hathaway, R., Barkey., M.: Fatigue Testing and Analysis: Theory and Practice. 1st edn. Elsevier Butterworth-Heinemann, Burlington (2004)

11. Shen, C.L., Wirshing, P.H., Cashman, G.T.: Design curve to characterize fatigue strength. J. Eng. Mater. Technol. 118, 535-541 (1996)

12. Williams, C.R., Lee, Y., Rilly, Y.T.: A practical method for statistical analysis of strain-life fatigue data. Int. J. Fatigue 25(5), 427-436 (2003)

13. Dieter, G.E., Bacon, D.: Mechanical Metallurgy. 3rd edn. McGraw-Hill Book Co., Singapore (1988)

Open Access This chapter is licensed under the terms of the Creative Commons Attribution 4.0 International License (http://creativecommons.org/licenses/by/4.0/), which permits use, sharing, adaptation, distribution and reproduction in any medium or format, as long as you give appropriate credit to the original author(s) and the source, provide a link to the Creative Commons license and indicate if changes were made.

The images or other third party material in this chapter are included in the chapter's Creative Commons license, unless indicated otherwise in a credit line to the material. If material is not included in the chapter's Creative Commons license and your intended use is not permitted by statutory regulation or exceeds the permitted use, you will need to obtain permission directly from the copyright holder.

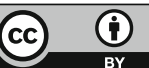

